International Journal of Advanced Studies in Humanities and Social Science (IJASHSS)

Available online at http://www.ijashss.com

Volume 8, Issue 1 (2019) pp. 61-70

Original Article

\title{
Identifying Causes of Confusion for Parents of Children with Developmental Disorders-Nervous
}

\author{
Farbod Mofidi' ${ }^{1}$, Hassan Ahadi' ${ }^{1}$, Farhad Jomehry'2, Mahdi Rahgozar ${ }^{3}$, \\ Ne'matollah Babaei ${ }^{3}$ \\ ${ }^{1}$ Department of Health Psychology, Karaj Branch, Islamic Azad University, Karaj, Iran \\ 2Department of Psychology, Allameh Tabatabaii University, Tehran, Iran \\ ${ }^{3}$ Department of Psychology, Social Welfare and Rehabilitation Sciences University, Tehran, \\ Iran
}

Received: 24 October 2018, Revised: 05 December 2018, Accepted: 20 December 2018

\begin{abstract}
This study was performed and aimed to identify the causes and types of confusion in the treatment of parents of children with developmental disorders. The questionnaire was designed to investigate the causes of confusion appropriate for our aim was completed by 350 parents of children who were purposefully selected. The obtained data were analyzed by factor analysis and then the relationship between each factor and the amount of confusion using log-linear analysis. The results indicate that factors such as control, beliefs, interaction of therapist - client and access to confusion treatment the type of internal special and factors such as beliefs, social- cognitive factors and interaction of therapist - client can predict the delay referring to the therapist. The investigation determined the greatest cause of confusion was socialcognitive factor, and minimum is commitment factor. Confusion is result of a combination of individual and environmental factors that confirms the need for individual counseling with parents at the same time informing health officials to help families of children with developmental disorder.
\end{abstract}

Keywords: Developmental Disorders, Compliance, Treatment, Causes Of Confusion.

\section{Introduction}

Confusion is a concept derived from the compliance and in this study; it means frequent reference of parents of children with developmental disorders to multiple experts; multiple specialties for its delay in diagnosis or treatment, and referral to start of treatment and rehabilitation process after the diagnosis.
It is assumed that some sort of confusion is the failure to compliance doctor's orders. This implies that patient non-adherence to program recommendations to forget or ignore or do not understand it properly and therefore it does not act right (Demateo, translated Kaviani, 2009). Researchers and experts believe that health field for 
the different causes for this confusion that can result from poor compliance of these factors:

Factors related to the patient's selfconcept, including self-efficacy, knowledge, intent, and etc.

Factors related to treatment, including the frequency and amount of drug use and treatment duration.

Economic and social factors such as social isolation and treatment costs.

Factors associated with conditions such as depression and associated symptoms of illness.

Factors associated with the health care team, such as the quality of communication and trust between the patient and health center staff (Sabata, 2003). Also it is shown that a combination of different factors such as age, severity of illness and other problems associated with disease take a part in the pursuit of treatment (Clarke et al., 2009).

Pirls and Jarata's studies (2000) suggest that other factors that may cause for patient confusion arguably include:

1) Factors related to relationship between the patient and physician: 2) Factors related to the health system, 3) Factors related to primary health care workers: 4) Factors related professionals. Also perceived severity of the health problem, perceived susceptibility to a disease, stimulants, demographic factors such as age, gender, race, ethnicity, and psychosocial factors such as social class characteristics and individual psychological and social pressure ... Affect the perceived risk of illness or injury. (Sarafino, translated Abhari, 2006).

A research reviewed the relationship between individual beliefs about health, health information, medical self-records. Multiple factor analysis of variables associated with reporting weak results, showed negative beliefs about treatment, age, and feelings of low selfcontrol in the treatment of hyperlipidemia. Also there was no correlation between health information beliefs and compliance (Gatti et al., 2009). Cognitive factors such as attention, memory, perception and beliefs also play a role in the formation of health behavior. Other factors arise from non-compliance in Bayn's research (1977) has shown that $37 \%$ of patients were unable to remember the name of the drug and $25 \%$ for the duration of their treatment; And Kreechton and colleagues (1978) discovered that 22\% of patients have forgotten, the recommended treatment plan. (Quoted by Curtis, 2000, translated Sohrabi, 2000). When the potential symptoms of the disease appear, proceed to early treatment, will be essential. Delay in treatment, according to the time interval between the first symptoms and the disease is when someone is attempting to use medical care. Other factors can be noted that the study treatment programs of Deelorio et al. (2006) are given in the appropriate doses of multiple drugs (drug levels) and different schedules can be far more difficult than programming the simpler treatment (Quoted by Boogar Rahimian, 2010).

Atrob and colleagues (1991), Gross and colleagues (1982) and Maand and colleagues (1993) believe when a person with chronic illnesses, is a child, the problem becomes more complicated if the child could not be firstly to recognize the importance of diagnosis and treatment and treat so he will be confused. Second, children often do not 
follow their treatment plan and family should be more involved treatment process. The complex interaction and interdependence between parent and child can reduce the issue of compliance with recommended treatment (Quoted by Sadoughi, Tamannayi Far, 2010). The relationship between patient and physician partially compromise the patient's expectations and fulfill it by the doctor. The researches of Kura and Colleague (1988), D. Lynch Miv and Cope (1985), Sanchez - Mengay, Heedroz and Cummings (1992) have shown that people in their assessment gave the highest score to the doctor or dentist friendly manner. As a human being, give attention to them, empathize with their feelings and show interest in them, they created a sense of confidence and encouragement and calm and in control face. (Quoting Sarafynv, 2002, translated Abhari et al., 2004). Researches of Di Matteo, (1985); Garity, (1981), Lee (1982) has shown that people who have more appropriate with their physician relationship are more likely to follow his medical advice. (Quoted Sarafino, 2002, translated Abhari et al., 2006). Other factors cited in this case are social factors. Messages we take from friends, family and health professionals will affect noticing of the problem and the action. In chronic diseases such as diabetes and destructive social support plays an important role in psychological adjustment and adherence to therapy plays (Sarafynv, 2002, translated Abhari et al., 2006). In chronic and destructive diseases such as diabetes social support plays an important role in psychological adjustment and adherence to therapy (Sarafino, 2002, translated Abhari et al., 2006). In a study in 2006 was conducted by Shahab Jahanlo and colleagues examined the relationship between quality of life and glycemic control, the results of this study, showed the relationship between the physical, social, and environmental and physical, psychological and environmental aspect and social, psychological aspect. It seems that tangible or instrumental support; informational support could have an important role in adherence (Sadducee, Tmannayi Far, 2009). Parents who have children with pervasive developmental disabilities are, in addition to being the parent of the nature of the task itself says the team anxiety Confused and disoriented from being why and how to deal with children with developmental disabilities are pervasive (Brezensky, 2004). Children born to such a push factor for families is a cause of concern for family adaptation (Seif Naraghi and Naderi, 2000). Developmental disorders of the nervous group of conditions that are considered during development and prior to entering school at an early age will rise and developmental defects, malfunctions or failures resulting in personal, social, academic or performance are specified (Fifth Diagnostic and Statistical Manual of Mental Disorders, 2013). In sum, given that the issue of non-adherence to medication (which in this study is equivalent to the term wandering considered) has been especially in group of children with developmental disorders that require long-term treatment and rehabilitation programs, But no research found Confusion of their parents. Despite the lack of evidence in adherence to medical and rehabilitation, on various aspects of physical, psychological, social and cognitive effects on children and the future of children and families, researchers in this study regarding the cultural aspects of 
the decision to identify causes and types confusion of and the relationship between these two factors were to change, it must devise ways to help these parents.

\section{Methodology}

Study design is applied research primarily because it seeks to use information obtained from research to respond to the problems of diagnosis and treatment of children with developmental disorders. As followed research method to answer the research question, the type of applied research is descriptive and correlation. The statistical population consists of all parents of children with developmental disorders, referred to treatment and rehabilitation centers in Karaj. The study samples are 350 families with children with developmental disorders. Most questionnaires completed by the mothers and the direct guidance and supervision of interviewers, and finally, after discarding incomplete questionnaires, 329 questionnaires were analyzed, the number entered. Sampling for this study is purposive sampling. To conduct this study a questionnaire of parent's confusion causes was designed and validated. Then, After passing the administrative process, a list of rehabilitation centers and clinics providing health services to children with developmental disorders of the Alborz Province was prepared from Welfare (Behzisti) Organization so, data will be collected to determine statistical population is selected by Purposeful sampling at stage of sampling from the centers of the study sample. After coordination with clinic managers and questionnaires carried out. For data analysis in SPSS 20 software indices of statistic, factor analysis of a questionnaire designed to identify the principal components analysis and in order to investigate the relationship between the causes of confusion and confusion types the loglinear analysis was used.

\section{Research Tools}

Researcher made questionnaire consists of two parts. The first part of the questions is characterized by types of confusion. Time difference problem diagnosis and onset of treatment or delayed-type of confusion total refer to a variety of expertise and treatments or confusion in type of among specialties (between different fields of medicine and rehabilitation)The total number of visits to specialists or confusion in type of intra specialties Replacement doctor or therapist in one field) In the second part is a questionnaire with 46 items and 6 factors (control, lack of commitment, belief, and social factors cognitive, interactive therapist - client access to treatment). Each question in this questionnaire would be graded on a scale of 5 scores from strongly disagree (1) to strongly agree (5). The Cronbach's alpha for control is equal to $0 / 81$, no commitment $0 / 74$, believe $0 / 85$, social cognitive, $0 / 84$, the interaction between the therapist - client, $0 / 83$, access to treatment $0 / 74$ of the total alpha is equal to $0 / 80$.

\section{Results}

According to demographic information the age of participated children in this study was $6 / 5 y e a r s, 1 / 60$ percent male, and most were first child. The results have shown that the majority of nonconsanguineous marriage and had no family history and know themselves in the middle class economically. In the descriptive findings section it was 64 | Page 
shown referring to a neurologist or pediatrician have had the highest frequency. But the parents who have been referred to various specialists in two expertise of pediatric and neurology it is seen a significant number of 3 Replacement specializes, that means more confusion is seen in the specialties of neurology and pediatrics. Also 55/6\% of parents with at least have visited two or three therapists to treat their children. But $28 / 9 \%$ of parents have experienced more than 3 therapists with their child's to treat. About the difference between the time of initial diagnosis and treatment, $22 / 5 \%$ of the subjects at the time of diagnosis, have started the treatments in total, more than the sample group have started their treatment with delay.

Table 1. Descriptive Statistics Types of Confusion

\begin{tabular}{ccccc}
\hline Mode & Mean & Maximum & Minimum & Types of confusion \\
\hline 7 & 13.81 & 64 & - & Intra specialty \\
9 & 8.27 & 21 & - & $\begin{array}{c}\text { Between specialty } \\
\text { Changing the specialty } \\
0\end{array}$ \\
& 15.20 & 106 & 0 & Delay \\
\hline
\end{tabular}

Table 2. The relationship between the causes of confusion and confusion types

\begin{tabular}{|c|c|c|c|c|c|c|c|c|}
\hline $\begin{array}{l}\text { Access to } \\
\text { treatmen }\end{array}$ & $\begin{array}{l}\text { Therapist- } \\
\text { client } \\
\text { ineraction }\end{array}$ & $\begin{array}{l}\text { Social- } \\
\text { cognitive }\end{array}$ & believe & $\begin{array}{c}\text { Non } \\
\text { commitment }\end{array}$ & control & & $\begin{array}{l}\text { causes of } \\
\text { confusion }\end{array}$ & \\
\hline $20.70 \%$ & $18.00 \%$ & $15.30 \%$ & $16.20 \%$ & $9.90 \%$ & $19.80 \%$ & percentage & $\begin{array}{c}\text { Between } \\
\text { specialty } \\
\text { (Changing } \\
\text { the } \\
\text { specialty) }\end{array}$ & $\begin{array}{c}\text { confusion } \\
\text { types }\end{array}$ \\
\hline $17.50 \%$ & $17.50 \%$ & $9.50 \%$ & $30.20 \%$ & $9.50 \%$ & $15.90 \%$ & percentage & $\begin{array}{l}\text { Intra- } \\
\text { specialty } \\
\text { (changing } \\
\text { specialist } \\
\text { ) }\end{array}$ & \\
\hline $10.20 \%$ & $16.40 \%$ & $32.80 \%$ & $15.60 \%$ & $12.50 \%$ & $12.50 \%$ & percentage & Delay & \\
\hline $15.60 \%$ & $17.20 \%$ & $21.50 \%$ & $18.90 \%$ & $10.90 \%$ & $15.90 \%$ & percentage & Total & \\
\hline
\end{tabular}

According to this table it can be seen replacing 64 times intra specialty the expertise and 21 times various specialties.

Given that in Table 2, we are sure about the relationship between the two variables of the model that presented in form of a diagram can be generalized.

\section{Subsidiary Findings}

The table above shows the most common cause of confusion in the studied sample is social-cognitive causes whilst predominant cause of confusion forms $21 / 5 \%$ of the samples examined the social causes - cognitive. Later, in order cause belief, to engage the therapist - client control, have access to treatment. The reason for the confusion is the smallest subjects as well as a lack of commitment. Figure 1 illustrates the relationship between the two variables of different types of confusion and causes of confusion as follows: 


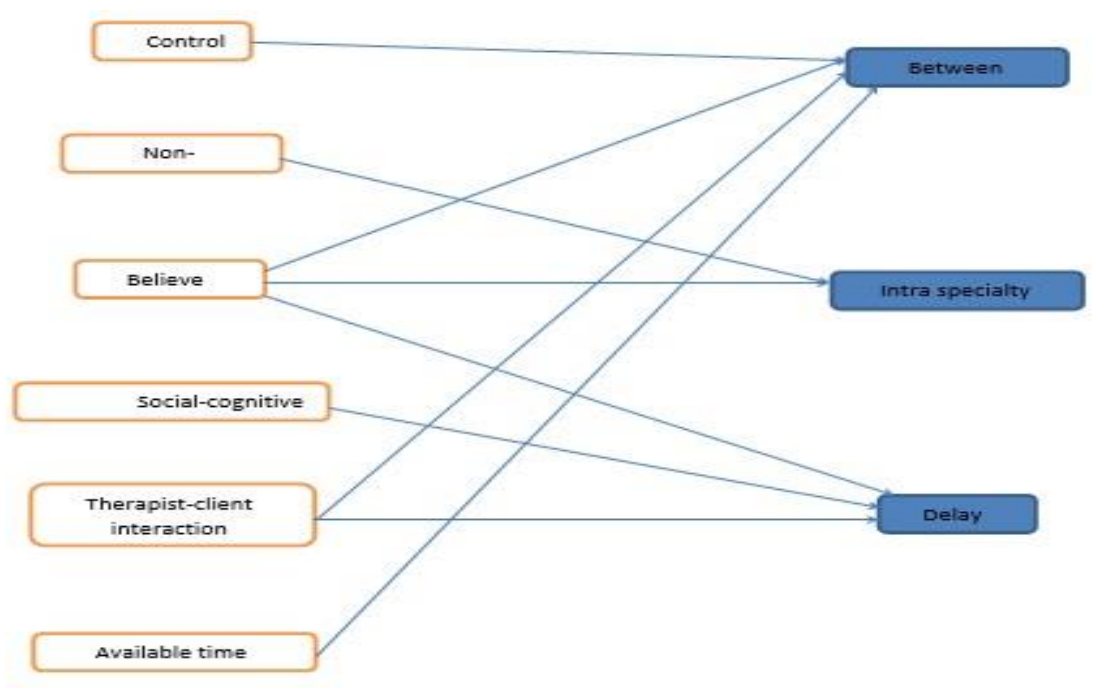

Figure 1. Relations between different levels of variables

\section{Conclusion}

This study aimed to identify the causes and extent of confusion of parents of children with developmental disorders. To achieve this objective, questionnaires were distributed among the parents of children with developmental disorders, were analyzed and the results are discussed as follows.

People who confused between different specialties are more influenced diagnostic and therapeutic agents to treat, control and interact with the therapist - client and the noncommitment factor has the least impact on the this group's confusion.

People who confused between different specialties and refer to different therapists during the treatment process are more influenced by believe factor and non-commitment and social - cognitive factors have minimal impact on the confusion of this Group.

Parents who delay between their children diagnosis and treatment are more influenced by social-cognitive factors and understanding and managing access to treatment has minimal impact on this group's confusion.

About $37 \%$ of the sample suffered from confusion of between specializations. After that $42 \%$ of the samples have been confused by type of delaying. Confusion of around 21\% of the samples also has experienced the confusion of between specialists.

In expression between different types of confusion of and causes of confusion it should be said:

People who the majority causes of their confusion has been control, have been more suffered from confusion of between specialties (replace the expertise).

People who lack of commitment were the predominant cause of their confusion they more have been into between specialties type of confusion.

It cannot be said that those who believe they have been the predominant cause confusion, confusion Quandaries have experienced any more than any other, that these people have experienced the same kind of confusion. 
Social- cognitive causes confusion are more conducive to confusion delay in type.

Confusion which is the result of interaction between therapist and client is further people experience confusion the type of between specialties (switching specialties), and delay.

People who most of their confusion more have been access to confusion have been affected between the types of specialization (replace the expertise).

\section{Discussion}

Results from the study effect of causes such as control, lack of commitment, belief, social factors - cognitive, interactions therapist - client and access treatment confusion among parents and medical professionals of different disciplines, medical practitioners or multiple substitutions in a string of delay to the start of treatment is consistent with research results of Demateo and colleagues (1993) which The number of patient visits per week for clinics, schedule frequent follow-up, fitness training with the needs of patients, the number of tests required by the severity of the disease, and stress-related health practitioner expertise that were considered important in the following. Videjanrako, Gompelman, Deejekroz et al. (2009) these factors include poverty, social protection, the negative attitude of the therapist, treatment and quality of the treatment.

Furthermore the social -cognitive factors which deal with believe and accept that the question of treatment, the therapist is consistent with researches of Pourtsa, Frankisb and Banda (2000), Horn Vinah (1999), Horn (1993), size and colleagues (2009) and
Afkhamzadeh Ebrahimi Nasr Esfahani Thaqafi (2000).

In addition, Parvizi, thereby, Vamqy and Foroughan (2000) on the relationship between trust and satisfaction of the patients with better compliance with the findings of the present study are in agreement. In order to align the mode of interactions the therapist and the client in line is consistent with research Reno and Mac, Gareth (2006).

From the results obtained it is concluded that in the sense of being control of their parents is confusion between specialties this means that parents follow their child a sense of control over the situation by most referred to experts in different fields. Positive effects of sense of control over patients' disease has been nearly confirmed For example, having a sense of control over the disease, in treatment patients who have had open-heart surgery is effective, Sense of control in these patients understand the disease positively and negatively associated with the severity of symptoms and the whole feeling of control is a positive impact for patients (Dojinvsky, Namoska and Restovska, ...). It seems to have replaced a few therapists can help ensure the diagnosis or therapy strategy; but, of course, especially in the rehabilitation process repeated switching would not be in favor of the parents and the child why replacing any therapist who spent time and energy to build relationships and promote each treatment plan will go to waste treatment sessions. Factor of parent s' lack of commitment can have relationship with sufficient sense (National Information Center for Children and Young America, 2003). 
The next factor in this study was identified as a cause of confusion for parents it is called believe. Individual or cultural beliefs about illness, how they should be treated, and what people should do to help him is the lack of an effective compliance (National Center for Disease Control and Prevention, 1999). Moreover, health beliefs and understanding of the health effects of non-compliance can also be effective (Martin, William, Heskar and Demateo, 2005). The belief of non-belief or suspicion to diagnosis and differential diagnosis or treatment is parental expectations. Confusion can be caused by parents in relation to children with special needs and their reactions to their child's disability. Another factor determining the confusion of socialcognitive factor which is defined is consisting reasons. Social and psychological factors, including family support and misdirection, it sometimes also referred to several studies and above these factors effectively and correctly follow medical instructions can be effective. The impact of the research Rahimian, gospel, immigrants and fortune-friendly (2012) and Wilkinson and Marmot (2003) is confirmed. These researchers believe that social support is an important factor in health and social support plays an important role as a source of emotional and health needs are met. Beleski (1984) believes appropriate social support to parents feel good about themselves and gives them their daily performance. In addition, social support of parents for direct or indirect impact on the lives their children to prepare, and the support system of family child care have an important role in child development (Harkns and Super, 1995).
Physician - patient relationship as one of the relevant factors affecting adherence to treatment for patients. Activities are an integral component in physicians' communication skills (Zali, 2009). In another study which was conducted in this field the results showed that the quality of the relation and trust between health care providers and patient has a relationship with adherence to treatment and will increase that (Morphy, 2004). In a research titled as nonconformity of treatment and physician - patient relation was conducted over 45,700 patients from all over Europe the results achieved that the individual's perception about the methods of therapy and medication is prescribed and how to better treat and can predict the patients adherence (Steraopolo, 2010). The other result obtained in this study was that $82 / 6 \%$ of subjects participating in the study have been reported no family history of developmental disorders in their children. Which this finding is not consistent with other researchers' findings who believe that genetics can have an important role in many nervous development disorders (Fifth Diagnosis Statistics manual, developmental disorders, 2013). These findings appear to be contradict of the reasons include remaining disorders unknown such as specific language impairment, hyperactivity, and learning problems in children of earlier generations. Due to the unknown nature of the problem or diagnosis, parents are not aware of their child's genetic history or even be the cause of these disorders can be complex and multi-faceted nature of psychological risk environment along cause of genetic and the life And of course cases such as alcohol, cigarettes 
and drugs of parents which can be the cause of developmental disorders should not be ignored (Nij, 2006, Ding et al, 2002, Topper, Holems, Polten, Hartington, 1999).

Therefore, these findings can be explained such that a) in a sample of adult ethnic disturbances have not been diagnosed. B) The cause is really a combination of non-genetic and unknown factors. At the end, dear Parents and rehabilitation centers in the city of Karaj who helped us in this study would be thanked.

\section{References}

A publication of the National Information Center for Children and Youth with Disabilities .2003. Parenting a Child with Special Needs.

American Psychiatric Association (2013). Diagnostic and Statistical Manual of Mental Disorders, DSM-5.

Belsky, J. (1984). The determinants of parenting: A process model. Child Development, 55, 83-96.

Berzonsky M. (2004). Identity style, parental authority, and identity commitment. Journal of Youth and Adolescence, 33(3), 213-220.

Centers for disease control and prevention. (1999). Patient adherence to tuberculosis treatment. Public Health Practice Program Office Division of Media and Training Services. Atlanta, Georgia

Clark, R.E., Weir, S., Ouellett,e R.A., Zhang, J., and Baxter, J.D. (2009). Beyond Health Plans: Behavioral Health Disorders and Quality of Diabetes, Journal of Antimicrobial Chemotherapy, 66(7), 657-64.
DiMatteo, M.R., Sherbourne, C.D., Hays, R.D., Ordway, L., Kravitz, R.L., McGlynn E.A., Kaplan, S., and Rogers, W.H.(1993). Physicians' characteristics influence patients' adherence to medical treatment: results from the Medical Outcomes Study. Health Psychol, 12(2), 93-102.

Ding, Y.C., H.C. Chi, D.L. Grady, A. Morishima, J.R. Kidd, K.K. Kidd, P. Flodman, M.A. Spence, S. Schuck, J.M. Swanson, Y.P. Zhang, and R.K. Moyzis. (2002). Evidence of positive selection acting at the human dopamine receptor D4 gene locus. Proceedings of the National Academy of Sciences, 99 (1): 309-14.

Dojcinovski, I., Naumoska, L.J, Ristovska, F. FEELING OF CONTROL OVER THE ILLNESS AND POSSIBLE HEALTH BENEFITS IN CARDIAC SURGERY PATIENTS. SPECIAL HOSPITAL FILIP VTORI Skopje, Macedonia; www.cardiosurgery.com.mk

Gatti, M.E., Jacobson, K.L., Gazmararian, J.A., Schmotzer, B., and Kripalani, S. (2009). Relationships between beliefs about medications and adherence. School of Medicine, George-town University, Washington, DC, USA. Unpublished.

Harkness, S. and Super, C. (1995). Culture and parenting. In M.H. Bornstein (Ed.), Handbook of parenting: Volume 2. Biology and ecology of parenting (pp. 211-234). Mahwah, NJ: Erlbaum.

Horne, R. (1993). Compliance, adherence, and concordance: One to be taken daily reflections on nonadherence (non-compliance). J Soc Adm Pharm, 10(2), 150-6. 
Horne, R. and Weinman, J. (1999). Patient's beliefs about prescribed medicines and their role in adherence to treatment in chronic physical illness. $J$ Psychoses Res, 47(6), 555-67.

Leslie R Martin, Summer L Williams, Kelly B Haskard, and M Robin DiMatteo (2005). The challenge of patient adherence. Ther Clin Risk Manag. 1(3), 189-199.

Murphy, D.A., Marelich, W.D., Hoffman, D., Steers WN (2004). Predictors of antiretroviral adherence. AIDS Care; 16(4), 471-84.

Nigg, J.T. (2006). What Causes ADHD? Understanding What Goes Wrong and Why. New York: The Guilford Press.

Pérez de Agreda, A., Bordejé M.F., and De Jarauta, D.J. (2000). Factors related to inappropriate referral between primary and specialized care: qualitative study among primary care physicians. Gac Sanit, 14 (2), 122-30 .

Porteousa, T., Francisb, J., Bonda, C. (2000). Temporal stability of beliefs about medicines: Implications for optimizing adherence. Patient Educ

Couns; 79(2): 225 -30.
Reyno, S.M. and McGrath, P.J. (2006). Redactors of parent training efficacy for child externalizing behavior problems a meta-analytic review. Journal of Child Psychology and Psychiatry, 47(1), 99111.

Sabaté, E. (2003). Adherence to longterm therapies - evidence for action. Geneva: World Health Organization.

Stavropoulou, C. (2010). Non adhevence to medication and doctorpatient relationship. $J$ Patient Eduaction Counseling, 10 (9), 698-705.

Thapar, A., J. Holmes, K. Poulton, and R. Harrington. (1999). Genetic basis of attention deficit and hyperactivity. British Journal of Psychiatry, 174, 10511.

Widjanarko, B., Gompelman, M., Dijkers, M., et al. (2009). Factors that influence treatment adherence of tuberculosis patients living in Java, Indonesia. Patient Preference and Adherence, 3 .231-238.

Zali, M.K.(2009). The novel principle of doctor and patient relationship. Tehran: Research Ethics; [Persian]

How to cite this article: Farbod Mofidi, Hassan Ahadi, Farhad Jomehry, Mahdi Rahgozar, Ne'matollah Babaei, Identifying Causes of Confusion for Parents of Children with Developmental Disorders - Nervous. International Journal of Advanced Studies in Humanities and Social Science, 2019, 8(1), 61-70. http://www.ijashssjournal.com/article 84109.html 